\title{
Comparability of Raman Spectroscopic Configurations: A Large Scale Cross-Laboratory Study
}

Shuxia Guo, Claudia Beleites, Ute Neugebauer, Sara Abalde-Cela, Nils Kristian Afseth, Fatima Alsamad, Suresh Anand, Cuauhtemoc Araujo-Andrade, Sonja Aškrabić, Ertug Avci, Monica Baia, Malgorzata Baranska, Enrico Baria, Luis A. E. Batista de Carvalho, Philippe de Bettignies, Alois Bonifacio, Franck Bonnier, Eva Maria Brauchle, Hugh J. Byrne, Igor Chourpa, Riccardo Cicchi, Frederic Cuisinier, Mustafa Culha, Marcel Dahms, Catalina David, Ludovic Duponchel, Shiyamala Duraipandian, Samir F. El-Mashtoly, David I. Ellis, Gauthier Eppe, Guillaume Falgayrac, Ozren Gamulin, Benjamin Gardner, Peter Gardner, Klaus Gerwert, Evangelos J. Giamarellos-Bourboulis, Sveinbjorn Gizurarson, Marcin Gnyba, Royston Goodacre, Patrick Grysan, Orlando Guntinas-Lichius, Helga Helgadottir, Vlasta Mohaček Grošev, Catherine Kendall, Roman Kiselev, Micha Kölbach, Christoph Krafft, Sivashankar Krishnamoorthy, Patrick Kubryck, Bernhard Lendl, Pablo Loza-Alvarez, Fiona M. Lyng, Susanne Machill, Cedric Malherbe, Monica Marro, Maria Paula M. Marques, Ewelina Matuszyk, Carlo Francesco Morasso, Myriam Moreau, Howbeer Muhamadali, Valentina Mussi, Ioan Notingher, Marta Z. Pacia, Francesco S. Pavone, Guillaume Penel, Dennis Petersen, Olivier Piot, Julietta V. Rau, Marc Richter, Maria Krystyna Rybarczyk, Hamideh Salehi, Katja Schenke-Layland, Sebastian Schlücker, Markus Schosserer, Karin Schütze, Valter Sergo, Faris Sinjab, Janusz Smulko, Ganesh D. Sockalingum, Clara Stiebing, Nick Stone, Valérie Untereiner, Renzo Vanna, Karin Wieland, Jürgen Popp, and Thomas Bocklitz*

Cite This: Anal. Chem. 2020, 92, 15745-15756

Read Online

ACCESS | Lلll Metrics \& More | 回 Article Recommendations | Sl Supporting Information

ABSTRACT: The variable configuration of Raman spectroscopic platforms is one of the major obstacles in establishing Raman spectroscopy as a valuable physicochemical method within real-world scenarios such as clinical diagnostics. For such real world applications like diagnostic classification, the models should ideally be usable to predict

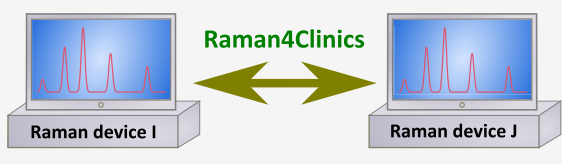
data from different setups. Whether it is done by training a rugged model with data from many setups or by a primary-replica strategy where models are developed on a 'primary' setup and the test data are generated on 'replicate' setups, this is only possible if the Raman spectra from different setups are consistent, reproducible, and comparable. However, Raman spectra can be highly sensitive to the measurement conditions, and they change from setup to setup even if the same samples are measured. Although increasingly recognized as an issue, the dependence of the Raman spectra on the instrumental configuration is far from being fully understood and great effort is needed to address the resulting spectral variations and to correct for them. To make the severity of the situation clear, we present a round robin experiment investigating the comparability of 35 Raman spectroscopic devices with different configurations in 15 institutes within seven European countries from the COST (European Cooperation in Science and Technology) action Raman4clinics. The experiment was developed in a fashion that allows various instrumental configurations ranging from highly confocal setups to fibre-optic based systems with different excitation wavelengths. We illustrate the spectral variations caused by the instrumental configurations from the perspectives of peak shifts, intensity variations, peak widths, and noise levels. We conclude this contribution with recommendations that may help to improve the inter-laboratory studies.

\section{INTRODUCTION}

Raman spectroscopy is known as a noninvasive, label-free technology with high selectivity, and thus, it has been increasingly applied to biological studies. ${ }^{1-3}$ These studies include forensics, ${ }^{4,5}$ diagnostics, ${ }^{6-9}$ metabolism research, ${ }^{10-12}$ microbiology, ${ }^{13,14}$ clinical pharmacology, ${ }^{15-17}$ and food sci-
Received: June 24, 2020

Accepted: November 2, 2020

Published: November 21, 2020 
Table 1. Number of Spectra for each Substance from each setup ${ }^{a}$

\begin{tabular}{|c|c|c|c|c|c|c|c|}
\hline setup ID & Wl./nm & $\# \mathrm{NeAr}$ & \#agar & \#gelatine & \#paracetamol & \#polystyrene & \#cyclohexane \\
\hline ID01 & 514 & 10 & & & 10 & 10 & 10 \\
\hline ID02 & 514 & & & 10 & 10 & & \\
\hline ID03 & 515 & 711 & & & 1053 & 709 & 361 \\
\hline ID04 & 532 & 200 & 10 & 300 & 30 & 300 & 100 \\
\hline ID05 & 532 & 151 & 135 & 116 & 121 & 11 & 177 \\
\hline ID06 & 532 & & & & & 20 & \\
\hline ID07 & 532 & 30 & 135 & 10 & 80 & 12 & \\
\hline ID08 & 532 & & & & & 120 & \\
\hline ID09 & 532 & & & 10 & & & \\
\hline ID10 & 532 & 11 & 12 & 12 & 13 & 11 & \\
\hline ID11 & 532 & 10 & 12 & 10 & & & \\
\hline ID12 & 532 & & 12 & 10 & 12 & & 12 \\
\hline ID13 & 532 & 200 & 70 & 190 & 10 & 100 & 200 \\
\hline ID14 & 532 & 1000 & 60 & 100 & 985 & 900 & 500 \\
\hline ID15 & 780 & 30 & 22 & & 30 & & \\
\hline ID16 & 785 & 99 & & 60 & 150 & 20 & \\
\hline ID17 & 785 & 10 & & 10 & 10 & 10 & 10 \\
\hline ID18 & 785 & & & & & & 30 \\
\hline ID19 & 785 & & & 10 & & & \\
\hline ID20 & 785 & 40 & & 100 & 40 & & 40 \\
\hline ID21 & 785 & 10 & 10 & 10 & 10 & 10 & 10 \\
\hline ID22 & 785 & 10 & 10 & 10 & 10 & 10 & \\
\hline ID23 & 785 & 12 & 11 & & 20 & 30 & 50 \\
\hline ID24 & 785 & & & 875 & 20 & & 50 \\
\hline \multicolumn{8}{|l|}{ ID25 } \\
\hline ID26 & 785 & 200 & 58 & 93 & 4139 & 208 & 50 \\
\hline ID27 & 785 & 100 & 100 & 100 & 100 & 100 & 100 \\
\hline ID28 & 785 & & 10 & & & & \\
\hline ID29 & 785 & 10 & & 10 & 10 & & 10 \\
\hline ID30 & 785 & 11 & 10 & 11 & 12 & 11 & \\
\hline ID31 & 785 & 251 & 76 & 348 & 164 & 151 & 26 \\
\hline ID32 & 785 & 10 & & & 10 & 10 & 10 \\
\hline ID33 & 785 & & & & & 601 & 814 \\
\hline ID34 & 785 & & 23 & 10 & 17 & & 14 \\
\hline ID35 & 785 & & & & & 21 & \\
\hline
\end{tabular}

ence. ${ }^{18,19}$ Most of these studies are yet at the proof-of-concept stage and are performed using a single or multiple similar Raman spectrometer(s). While the Raman spectra from any sample contain the vibrational fingerprint information of the molecules within the sample, they unfortunately also contain fingerprints of the analytical setup, e.g., Raman spectrometer, itself. ${ }^{20}$ Therefore, the same sample can lead to different Raman spectra if measured on multiple setups, in different conditions, or at different times. To be clear, we will henceforth refer to 'setup' as an indication of all measurement related effects, be these temporal drifts, variations of measurement conditions, or instrumental configurations (viz. laser sources, spectral resolution).

Subtle differences in the Raman setup can degrade the reproducibility of the Raman spectroscopic signals, which makes it almost impossible to quantitatively compare measurements from different setups, and this is highly detrimental to crosssetup data analysis. The setup-dependence is very likely to have a larger influence in biological applications in which the spectral contributions of interest can be very subtle in the acquired Raman signal and thus are more easily masked. If Raman spectroscopy is expected to be utilized in, e.g., clinical scenarios, it is likely that one or more 'primary' setups hold a large database of Raman spectral characteristics of, e.g., some disease states. Other Raman setups in different laboratories act as 'replicate' setups and send data to the 'primary' setup for testing against this 'primary' model/database. If the Raman setups are significantly different, these statistical models are likely to fail to correctly predict the newly measured samples on the 'replicate' setups. Building up individual statistical models for each setup could be a solution to this problem, but this is not likely to be acceptable in real-world scenarios, as the models would also be setupdependent and this would further hamper the generalization of Raman spectroscopy.

A more feasible and attractive approach is to remove the setup-induced spectral variations from the spectral 'database'. This is the task of pre-processing in general. The most straightforward way to remove the confounding influence of a setup is spectrometer calibration, including wavenumber and intensity corrections. ${ }^{20-22}$ Spectrometer calibration corrects for the influence of a setup on the Raman signals by retrieving the correct values from the measurements of known standard materials. In many cases, an interpolation is needed to deal with the different nominal spectral resolutions of different setups. ${ }^{23}$ The extent to which an instrument software takes care of the spectrometer calibration varies greatly across manufacturers; so 
does the possibility to access raw data, which allows the users to apply their own calibration functions from a known basis. Spectrometer calibration does decrease the setup-dependence but rarely removes it completely, due to the inaccuracy in the estimated instrumental response function. The remaining setupderived spectral variations can still negatively affect the reliability of data analysis. In addition, the standard materials used for calibration may differ across laboratories (especially for biological materials), which can introduce additional spectral variations among different setups.

In addition to robust spectrometer calibration, standard-free approaches such as warping methods in which the unwanted spectral variations are removed by aligning all spectra against a given reference spectrum can also be used. ${ }^{24}$ However, as a purely data-driven procedure, it does not necessarily give meaningful results, as these procedures may remove both sample- and setup-induced variations without distinction. In this context, methods based on bilinear modeling, such as replicate EMSC (extended multiplicative signal correction) and ASCA (ANOVA-simultaneous component analysis), make it possible to estimate the setup-induced spectral differences and remove them from the data while preserving the sample-induced variations. ${ }^{25-27}$ This seems to be a more reliable mechanism, although it requires the experiments to be well designed. Such a method is also limited if the sample- and setup-induced spectral variations are not independent of each other.

Considering all the issues stated above, setup-dependence is still a significant concern and a major challenge to Raman spectroscopy being translated into real-world applications. To investigate this analytical challenge, we designed a round robin experiment with researchers from approximately 50 European institutes, which was initiated within the COST action Raman4Clinics. Briefly, aliquots of the same samples were prepared in one partner laboratory and sent to other laboratories in which data were collected using Raman spectrometers (see Table S1) from various manufacturers, with various laser sources, different spectral resolutions (i.e., pixel size), and optical configurations ranging from highly confocal to fibreoptic probes (and hence different numerical apertures). We note that, of course, there can be spectral differences due to resonance excitation, and therefore, for the present study, we have chosen Raman setups and samples absent of resonance Raman contributions. In the end, data from 35 setups of 15 institutes were returned to a single laboratory for unified data processing and assessments. The comparability of the setups was evaluated from the perspectives of the peak positions, peak intensities, peak widths, and noise levels. We report these differences and provide recommendations for future cross-laboratory studies. Ultimately, the translation of Raman spectroscopy as a bioanalytical protocol to clinical applications will require regulatory certification, as per, for example, the European Medicine Agency "Guideline on bioanalytical method validation", which details requirements of accuracy and precision. ${ }^{28}$ We hope that this study will trigger more unified efforts from the Raman community to come to some harmonization on handling setup-dependence, for instance, rules of setup standardization, certification of devices to be used in clinics, etc. In the end, we hope to help establish Raman spectroscopy as a reliable tool for real-world applications.

\section{EXPERIMENTAL AND METHODS}

Raman Spectroscopy. Details of the experimental design are given in the Supporting Information, and the spectral data are available via an open data repository (https://doi.org/10. 5281/zenodo.4152953). Briefly, we received data from 35 setups for the analysis in this contribution. The information of Raman setups is summarized in Table S2, which includes the manufacturer, the source wavelength, and the nominal spectral resolution (i.e., pixel size). Raman spectra of the $\mathrm{NeAr}$ glow lamp and different substances were measured, including agar, gelatine, paracetamol, polystyrene, and cyclohexane. The number of replicate spectra obtained using each setup from the different substances is given in Table 1 . The spectral data from setup ID25 were excluded from the analysis, due to recording errors in the wavenumber axis (see Figure S1).

Spectral Pre-Processing. The spectra from the NeAr lamp were normalized with respect to their maxima without any additional processing. All spectra from the five substances (i.e., agar, gelatine, paracetamol, polystyrene, and cyclohexane) were subjected to the same pre-processing steps. The details can be found in the Supporting Information. Briefly, any cosmic spikes were removed via a comparison between every pair of Raman spectra of the same substance. A wavenumber calibration was performed based on the spectra of the standard material paracetamol. Thereafter, all spectra were interpolated to an equidistant wavenumber grid of $1 \mathrm{~cm}^{-1}$. This is followed by a sensitive nonlinear iterative peak $\left(\mathrm{SNIP}^{29}\right)$ clipping algorithm to remove any broad baseline artifacts. In the end, a vector normalization $\left(l_{2}\right.$ norm $)$ was performed. Note, a wavenumber calibration was conducted on 23 of the instruments where paracetamol measurements were reported.

Spectral Characterization. In order to assess any variations between the different setups, the pre-processed Raman spectra were analyzed to investigate the following four aspects: signal-tonoise ratio (SNR), peak shift, peak width, and specific peak ratios. These four metrics were chosen to benchmark analytical sensitivity, the reproducibility of peak positions, the spectral resolution, and the reproducibility of relative intensities of a measurement. We consider these four properties to be the most important for establishing whether there are variations between the different Raman platforms. The definition of these characteristics and their calculation are summarized in the following.

Signal-to-Noise Ratio (SNR). The SNR can be used to determine the lowest signal that can be reliably detected, by comparing an average signal to the noise level. Herein, the SNR was calculated in two ways using the samples of agar and gelatine, which featured the highest noise among all measured substances in this study. The mean spectra of these two substances from each measurement are given in Figures S2,S3, along with the standard deviation, as shadows. In the first case, we calculated the SNR for each spectrum individually as the ratio between the mean Raman intensity and the standard deviation of the estimated noise (see eq $(S 3.1)$ ). The noise $\left(I_{n}\right)$ was estimated as the difference between the spectrum and the output of a strong Savitzky-Golay (S-G) smoothing $(p=2, n=31)$ of the spectrum. ${ }^{30}$ Herein, the parameters $p$ and $n$ represent the degree of the polynomial function and the window size of the $S$ $G$ filter, respectively. An example of spectrum before and after smoothing together with the estimated noise is given in Figure S8. As the noise could be upper-biased for the spectral region with sharp Raman bands, the SNR tends to be under-estimated in this case. In the second case, we calculated the SNR as the ratio between the mean and standard variation of the Raman intensities at a given wavenumber over 10 randomly selected Raman spectra (see eq (S3.2)) in which the peak intensities of 


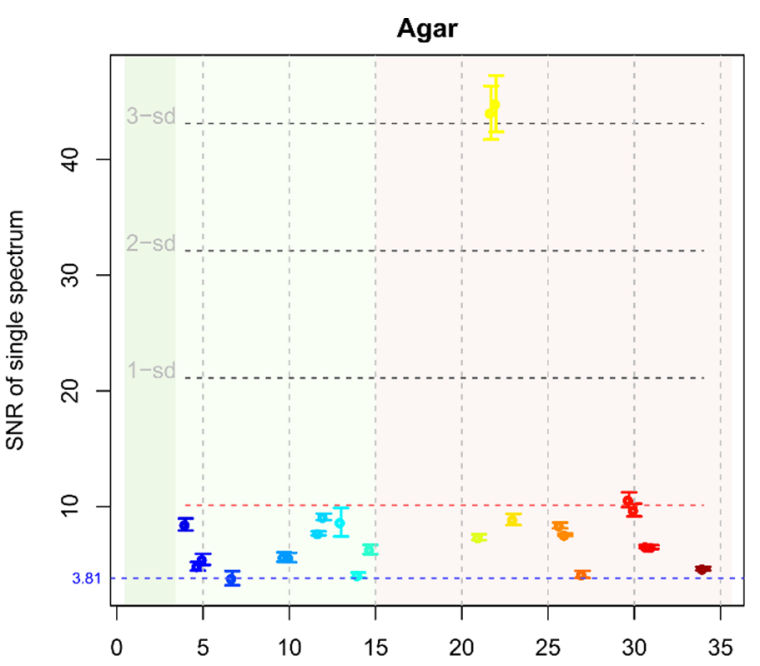

(a)

Setup ID

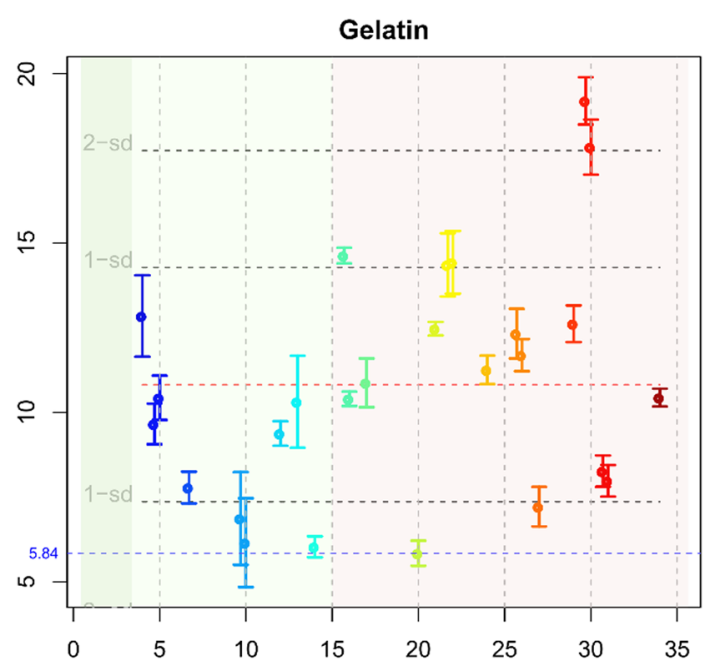

(b)

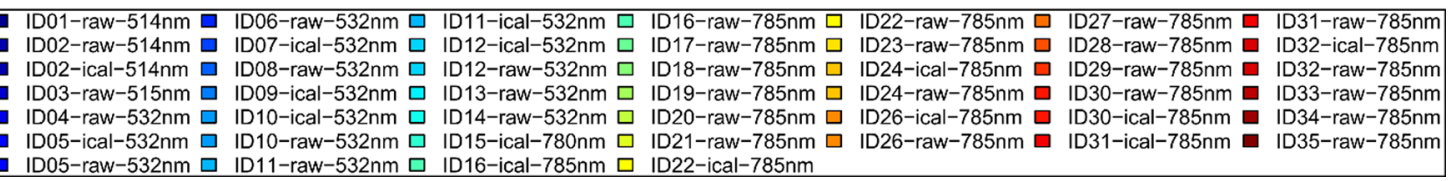

Figure 1. SNR of (a) agar and (b) gelatine: the color shades represent the setups with different source wavelengths. Each bar represents the measurement of a substance on a given setup. The bar contains the mean and standard deviation value of the SNR for each setup. The average and standard deviation of all setups are given using the red and gray dashed lines, respectively. The blue dashed line marks the minimal SNR of all setups. The identification of the setup, the type of data, and the source wavelength are given in the legend. In particular, the data types 'ical' and 'raw' denote the cases where the data were exported from the setup with (ical) and without (raw) intensity calibration, respectively.

the Raman bands around 846 and $1451 \mathrm{~cm}^{-1}$ were used for agar and gelatine, respectively. These two Raman bands were selected to be sufficiently intense and well separated from other bands.

Peak Shift. The peak shift characterizes the accuracy of the wavenumber axis ( $x$ axis, spectral axis) of a setup. It was defined as the deviation of a measured peak position from its theoretical position. To do so, we employed three substances having welldefined Raman bands: paracetamol, polystyrene, and cyclohexane. The mean spectra and standard deviations of the three substances for each setup are shown in Figures S4-S6 (a). The Raman bands used in our calculation are highlighted in Figures S4-S6 (b). Only the bands within the fingerprint region were considered, as the $\mathrm{CH}$ stretching region was not measured on all setups. To start, we fitted each of these Raman bands by a Gaussian peak according to eq 1 because Gaussian fits have been proven to work well for solid, powder, gel, or resin samples. ${ }^{31}$ The spectral region used for the fit was defined by $\tilde{\nu}_{0} \pm 10 \mathrm{~cm}^{-1}$, where $\tilde{\nu}_{0}$ denotes the theoretical peak position. The parameter $\mu$ of the resulting Gaussian peak was considered to be the measured peak position of the Raman band being fitted, and the peak shift of this Raman band was determined by $\Delta \tilde{\nu}=\mu-\tilde{\nu}_{0}$. The final result of one single spectrum was determined as the mean absolute value of all peak shifts from the multiple Raman bands within this spectrum, which indicates the mean absolute deviation in the wavenumber axis of a measurement.

$$
\mathrm{I}(\tilde{\nu})=\mathrm{A} \cdot \exp \left(-\frac{(\tilde{\nu}-\mu)^{2}}{2 \sigma^{2}}\right)
$$

Peak Width. The peak width is considered as a straightforward metric to characterize the spectral resolution of a given setup and can be benchmarked by the full-width-at-halfmaximum (FWHM). Herein, we employed the results of $\sigma$ from the peak fit described in the previous section and obtained the FWHM according to eq 2 . The calculation was performed on both the NeAr emission and the Raman spectra of the three substances, i.e., paracetamol, polystyrene, and cyclohexane. The measured $\mathrm{NeAr}$ emission on different setups is visualized in Figure S7. To make the determination more precise, the FWHM was only calculated for the best-defined peak in each case; i.e., no significant shoulder peaks exist, and the peak does not overlap with its neighboring peaks. According to this criterion, we employed the emission at 626.56 and $878.2 \mathrm{~nm}$ for the $\mathrm{NeAr}$ measurement with laser sources of $514 / 532$ and $785 \mathrm{~nm}$, respectively; the peaks at 1169,1602 , and $1028 \mathrm{~cm}^{-1}$ were used for paracetamol, polystyrene, and cyclohexane, respectively.

$$
\mathrm{FWHM}=2 \sigma \cdot \sqrt{2 \ln (2)}
$$

Peak Ratio. The peak ratio is defined as the ratio between the areas of two given Raman bands. It quantifies the consistency of the relative Raman intensity across setups, and it is an important criterion for a reproducible Raman spectroscopic data analysis, either quantitative or qualitative. In particular, the area of a given Raman band was calculated via a simple integration of the peak region according to eq 3 . We did not additionally subtract the baseline offset during this integration, as the spectra were already baseline corrected. The terms $k_{1}$ and $k_{\mathrm{r}}$ denote the start and the end index of the band. These values were determined automatically as the positions around a band where the Raman intensity starts to increase (i.e., $I\left(\tilde{\nu}_{k_{1}-1}\right)>I\left(\tilde{\nu}_{k_{1}}\right)$ ) or stops decreasing (i.e., $\left.I\left(\tilde{\nu}_{k_{\mathrm{r}}}\right)<I\left(\tilde{\nu}_{k_{\mathrm{r}}+1}\right)\right)$, respectively. We based our calculation on the spectra of cyclohexane and obtained three peak ratios for each spectrum: the area of the bands at 1028, 1266 , and $1444 \mathrm{~cm}^{-1}$ with respect to the area of the band at 801 $\mathrm{cm}^{-1}$. The four bands are well-defined and do not show 

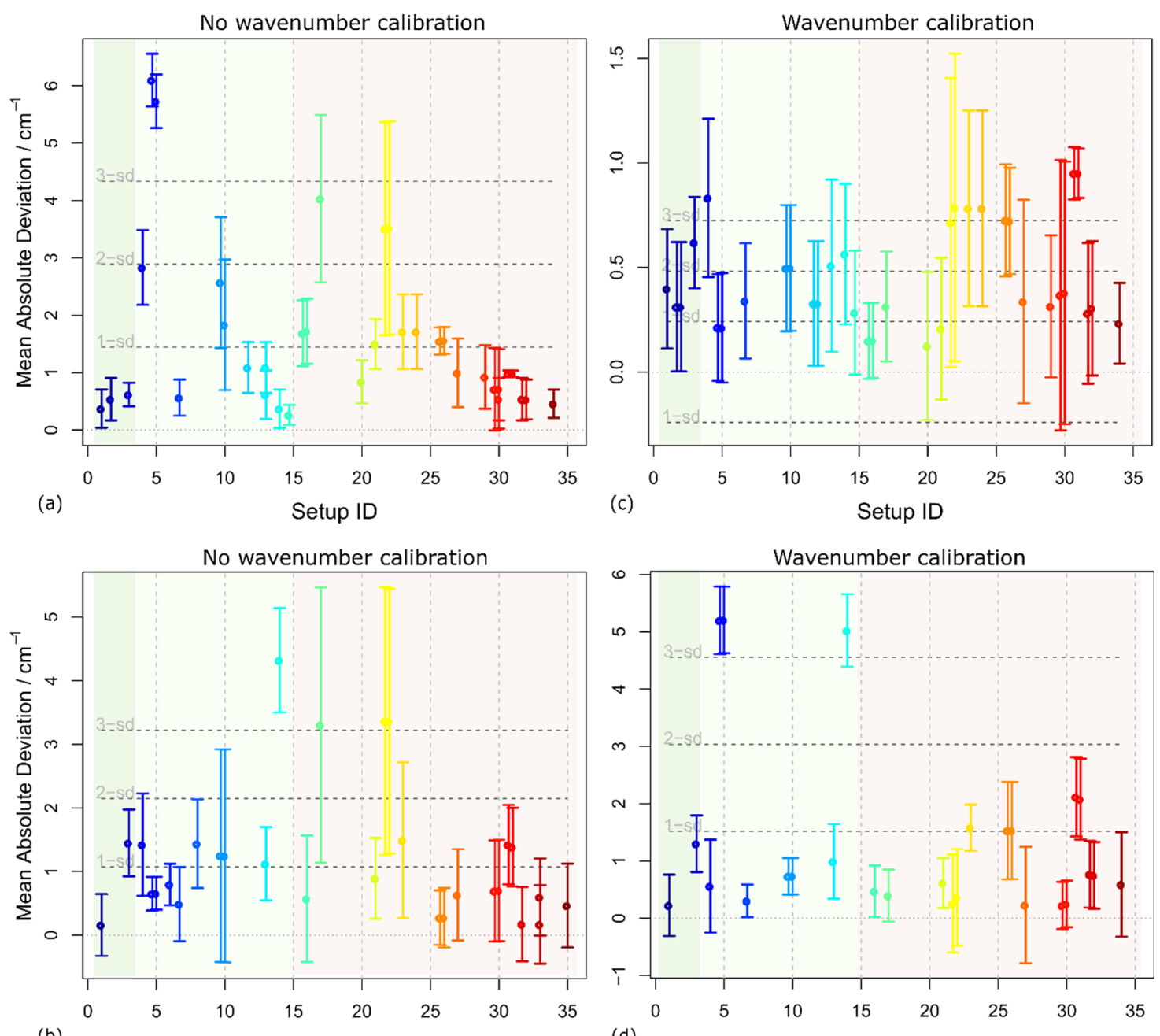

(b)

Setup ID

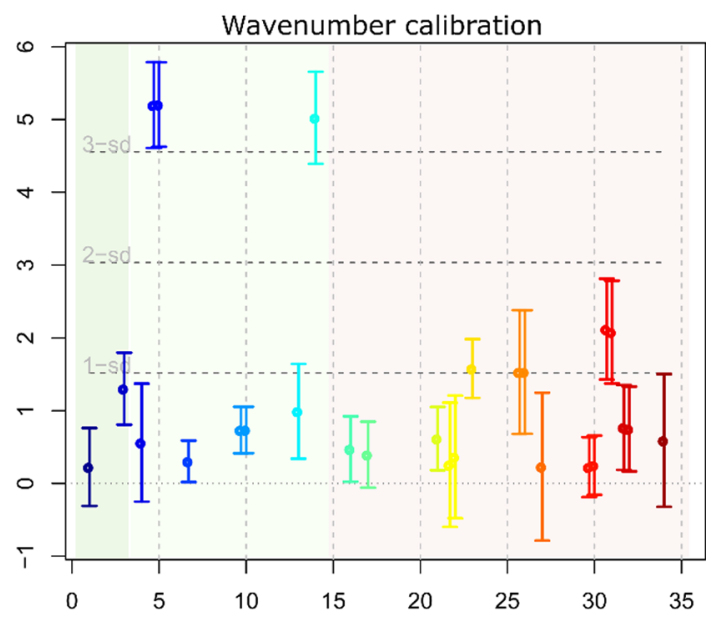

(d)

Setup ID

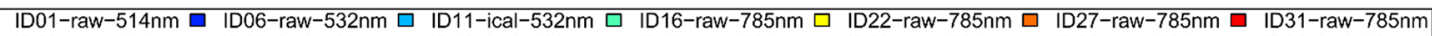

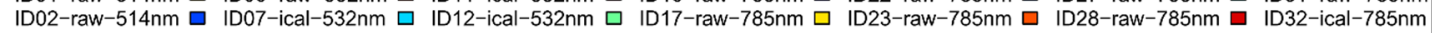

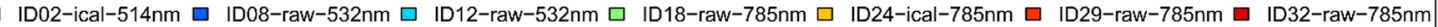

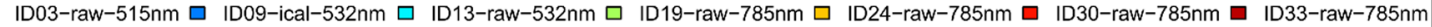

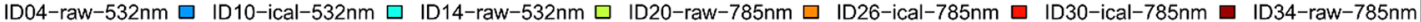
- ID05-ical-532nm ID10-raw-532nm ID15-ical-780 nm ID21-raw-785nm ID26-raw-785nm ID31-ical-785nm ID35-raw-785nm ID05-raw-532nm $\square$ ID11-raw-532nm $\square$ ID16-ical-785nm $\square$ ID22-ical-785nm

Figure 2. Results of peak shifts for (a, c) paracetamol and (b, d) polystyrene, without and with wavenumber calibration. The wavenumber calibration for polystyrene was only calculated if there is a paracetamol spectrum measured on the same setup. Therefore, a lower number of setups are shown for the calibrated results for polystyrene than without wavenumber calibration.

significant shoulders or overlap with neighboring bands, which helps to precisely estimate the peak area.

$$
A=\sum_{i=k_{1}}^{k_{\mathrm{r}}} I\left(\tilde{\nu}_{i}\right)
$$

\section{RESULTS AND DISCUSSION}

We present here the results of assessment of the abovementioned metrics calculated for different setups and substances. As our major focus in this study is the cross-setup comparability, we will mainly compare the results of different setups with each other but not specifically consider their reference values given in the literature. For such reference information, the interested readers are kindly referred to refs. $^{32-34}$

The SNR results using a single-spectrum and 10 replicate spectra are given in Figure 1 and Figure S9, respectively. The color shades represent setups with different source wavelengths. The meta-information of the datasets is provided in the legend, including the identification number (ID) of the setup, the type of the data, and the excitation wavelength for each measurement. The two data types, represented as 'raw' and 'ical', differed in whether an intensity calibration was done by the setup itself or not, respectively. In particular, the SNR of each measurement was plotted as a single bar containing the information of the mean and standard deviation over the multiple spectra from the same measurement (the number of replicates for each experimental setup is provided in Table 1). The red dashed line marks the global mean of the SNR over all measurements, while the gray dashed lines show 1 to 3 times the global standard deviation of SNR over all measurements. The minimal mean SNRs for agar and gelatine, given using the blue dashed line, are 3.8 and 5.8, respectively. That is to say, the SNR is sufficient for all setups, which is reasonable considering a preliminary SNR filtering during the data upload (see experimental design in the 
Supporting Information). This can be concluded from the SNR calculated from multiple spectra as well, as is shown in Figure S9.

The results of the peak shift calculations are plotted in Figure 2 and Figure S10, following the same structure as in Figure 1. It is implicit in the definition that the smaller the absolute value of the peak shift, the better the setup. An ideal measurement would give a zero peak shift; i.e., peaks from the measurement match perfectly with the theoretical values. As the ideal case is not easily achievable in reality, wavenumber calibration is important to minimize the peak shifts. To do so, we applied paracetamol as the standard material and calculated a calibration function based on the deviation between the measured and the theoretical positions for well-defined bands. This wavenumber calibration function was thereafter applied to the spectra of paracetamol, polystyrene, and cyclohexane. As shown in Figure 2 and Figure S10, the peak shifts were reduced closer to zero after the calibration, compared to before calibration. To make the conclusion clearer, we summarized the statistics of the peak shifts in Table 2 . Therein, $\Delta \overline{\tilde{\nu}}$ denotes the mean peak shift of

Table 2. Statistics of Peak Shifts, Summarized from the Results of Figure 2

\begin{tabular}{clccc} 
substance & & $\operatorname{mean}(\Delta \overline{\tilde{\nu}})$ & $s d$ & $\max \left(s d_{0}\right)$ \\
paracetamol & no calibration & $1.58 \mathrm{~cm}^{-1}$ & $1.44 \mathrm{~cm}^{-1}$ & $1.86 \mathrm{~cm}^{-1}$ \\
& calibrated & $0.45 \mathrm{~cm}^{-1}$ & $0.24 \mathrm{~cm}^{-1}$ & $0.73 \mathrm{~cm}^{-1}$ \\
polystyrene & no calibration & $1.20 \mathrm{~cm}^{-1}$ & $1.07 \mathrm{~cm}^{-1}$ & $2.16 \mathrm{~cm}^{-1}$ \\
& calibrated & $1.31 \mathrm{~cm}^{-1}$ & $1.52 \mathrm{~cm}^{-1}$ & $1.02 \mathrm{~cm}^{-1}$ \\
cyclohexane & no calibration & $1.33 \mathrm{~cm}^{-1}$ & $1.79 \mathrm{~cm}^{-1}$ & $1.37 \mathrm{~cm}^{-1}$ \\
& calibrated & $1.93 \mathrm{~cm}^{-1}$ & $2.16 \mathrm{~cm}^{-1}$ & $0.73 \mathrm{~cm}^{-1}$ \\
\hline
\end{tabular}

each setup. The factors $s d$ and $s d_{0}$ represent the standard deviation over all measurements and standard deviation for a single measurement, respectively. In particular, the decrease of $\Delta \overline{\tilde{\nu}}$ demonstrates an improved setup-independence after the wavenumber calibration, although we note that there are also setups that are (extremely) negatively affected (ID05; ID14) or not improved (ID31). A generally better reproducibility within the same measurement was observed after wavenumber calibration, evidenced by a reduced maximal $s d_{0}$. The increase in the mean and standard deviation of $\Delta \overline{\tilde{\nu}}$ after calibration for polystyrene and cyclohexane will be explained in the next paragraph.

As it is apparent in the figures, the improvement due to the wavenumber calibration was not the same for the three substances: it was the most significant for paracetamol but much less so for polystyrene and cyclohexane. As is shown in Table 2, the mean of $\Delta \overline{\tilde{\nu}}$ was decreased for paracetamol but not for the other two substances. This is easy to explain, as the calibration function of a setup was 'learnt' from the spectra of paracetamol and 'transferred' to the other substances. This function is supposed to capture best the peak shift of the Raman spectra of paracetamol and less so for the other substances. The performance of the wavenumber calibration can also be degraded due to other issues. For example, the peak shifts can increase after wavenumber calibration if the spectra of paracetamol measured on the specific instrument do not truly reflect the peak shift of spectra to be calibrated. This was seen for the setup 'ID05', for which the spectra of paracetamol exhibited significantly larger peak shifts than those of polystyrene and cyclohexane. The wavenumber calibration in this scenario introduced additional errors in the peak positions and led to increased peak shifts (Figure 2 and Figure S10). Another possibility that makes the wavenumber calibration invalid was seen from setup 'ID14', for which the peak shifts in paracetamol were much smaller than the shifts in the spectra of polystyrene and cyclohexane. Therefore, the calibration function could not represent the peak shifts well, and thus, the wavenumber calibration hardly reduced the peak shifts (Figure 2 and Figure S10). These two scenarios may happen if the standard material (e.g., paracetamol) and the samples to be calibrated (e.g.,

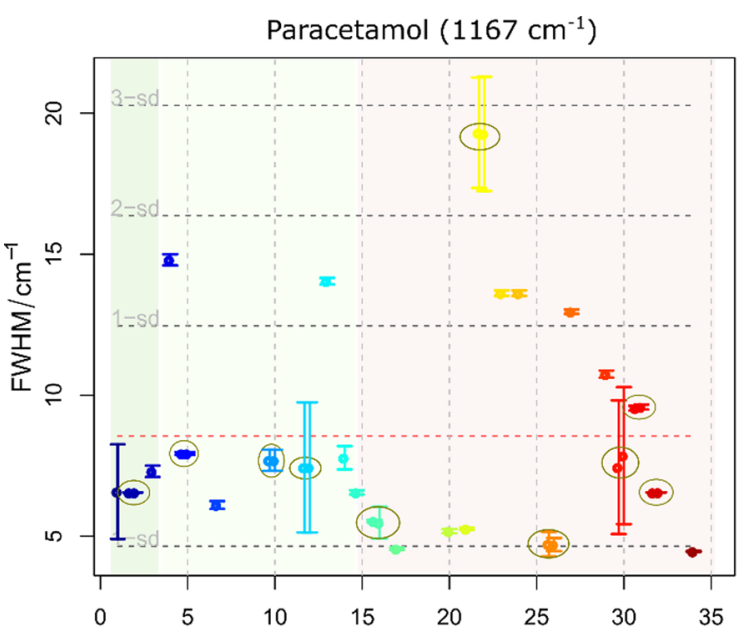

(a)

Setup ID

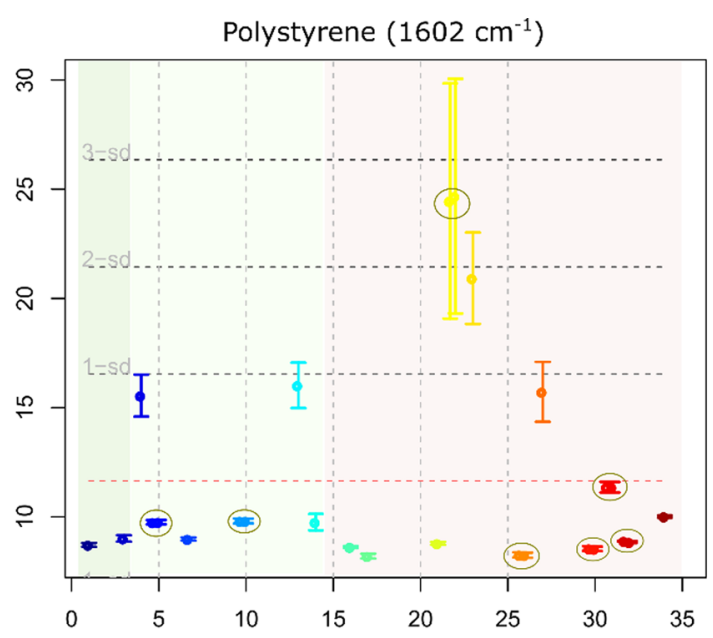

(b)

Setup ID

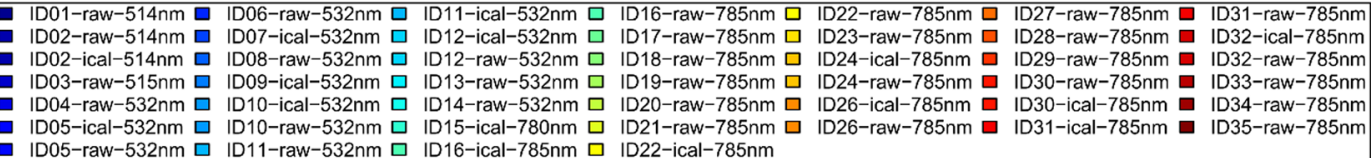

Figure 3. Results of FWHM calculated from (a) paracetamol and (b) polystyrene based on selected peaks. The setups from different source wavelengths were marked by the colored shades. Pairs of spectra with and without intensity calibration were highlighted by circles. The variations among different setups are clearly seen from the results. 


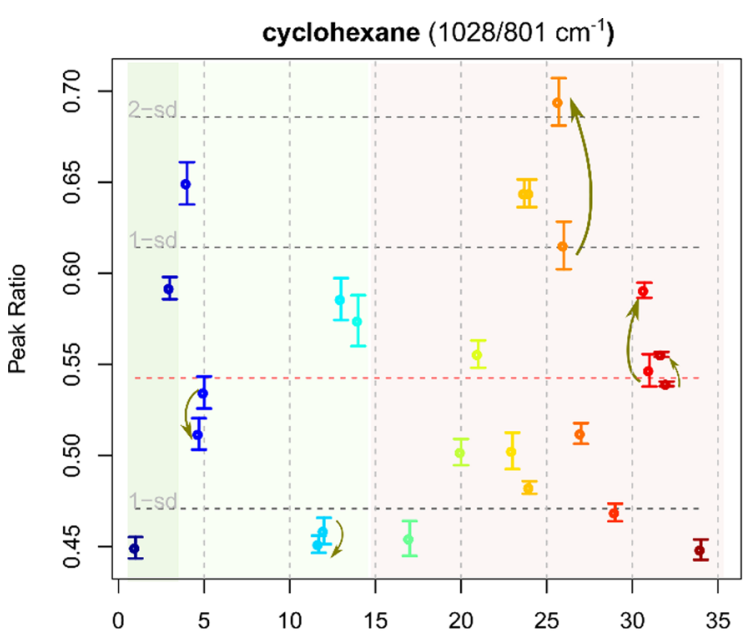

(a)

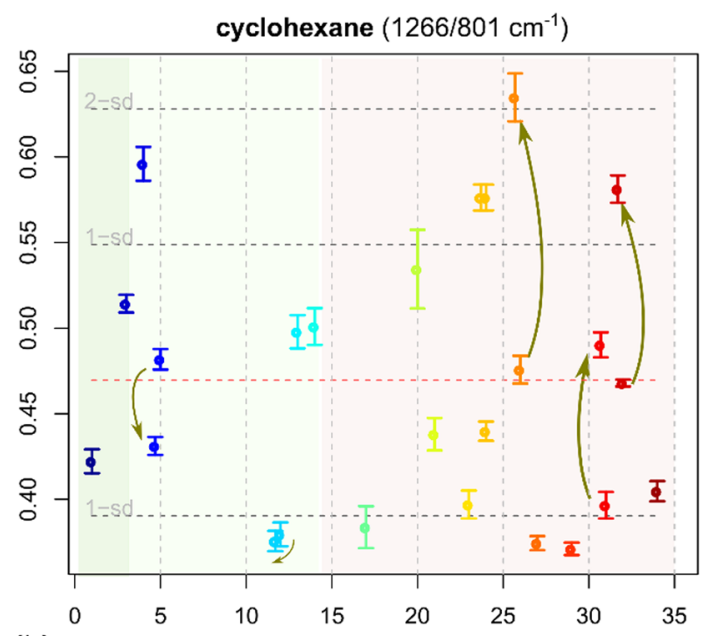

(b)

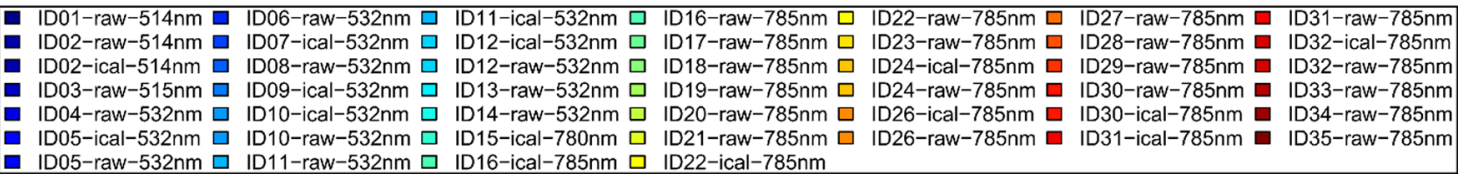

Figure 4. Ratios of peak intensities at (a) 1028 and (b) $1266 \mathrm{~cm}^{-1}$ to the peak intensity at $801 \mathrm{~cm}^{-1}$. The color shades represent the setups with different source wavelengths. The curved arrows in brown show the paired results without and with intensity calibration, pointing to the results with intensity calibration. It is clear that the peak ratios varied largely among measurements, and the setup intern intensity calibration does not improve the situation.

Table 3. Statistics of the Peak Ratios Summarized from Figure 4 and Figure S14 for the Setups with Different Source Wavelengths $(514,532 \text {, and } 785 \mathrm{Nm})^{a}$

\begin{tabular}{|c|c|c|c|c|c|c|c|c|c|}
\hline \multirow[b]{2}{*}{ raman bands $\left(\mathrm{cm}^{-1}\right)$} & \multicolumn{3}{|c|}{$\operatorname{mean}(\bar{r})$} & \multicolumn{3}{|c|}{$s d /$ mean $(\bar{r})$} & \multicolumn{3}{|c|}{$\max \left(s d_{0}\right) / \operatorname{mean}(\bar{r})$} \\
\hline & $514 \mathrm{~nm}$ & $532 \mathrm{~nm}$ & $785 \mathrm{~nm}$ & $514 \mathrm{~nm}$ & $532 \mathrm{~nm}$ & $785 \mathrm{~nm}$ & $514 \mathrm{~nm}$ & $532 \mathrm{~nm}$ & $785 \mathrm{~nm}$ \\
\hline $1028 / 801$ & 0.52 & 0.54 & 0.55 & 0.19 & 0.13 & 0.13 & 0.01 & 0.03 & 0.02 \\
\hline $1266 / 801$ & 0.47 & 0.47 & 0.47 & 0.14 & 0.17 & 0.18 & 0.02 & 0.02 & 0.05 \\
\hline $1444 / 801$ & 0.53 & 0.54 & 0.52 & 0.11 & 0.24 & 0.29 & 0.03 & 0.03 & 0.13 \\
\hline
\end{tabular}

${ }^{a_{T}}$ The terms mean $(\bar{r})$, $s d$, and $\max \left(s d_{0}\right)$ represent the global average, global standard deviation, and the maximal standard deviation of the peak ratios, respectively. The variations in the relative intensities tend to increase between two peaks with longer distance along the wavenumber axis.

polystyrene and cyclohexane in this study) are measured under different conditions. It could also be the case that the setup bears short-term instability, leading to variations among spectra of standard material and samples. This could be a significant concern in applying Raman spectroscopy. However, we will not go into details of this issue as we did not have sufficient data from this respect.

An additional interesting observation is that $s d$, which benchmarks the comparability across setups, was increased by wavenumber calibration for polystyrene and cyclohexane. There are two likely reasons, to our assessment. First, the two cases with improper calibration as mentioned previously contributed to the increase in $s d$. Second, the variations in the spectra of paracetamol may be passed to the calibration procedure and add to the variation in the calibrated spectra. With all these observations, it is obvious and fair to say that the peak shifts remain a significant issue for the cross-setup reproducibility in Raman spectroscopy. Wavenumber calibration, as is already shown in a previous report, ${ }^{21}$ cannot completely remove the setup-induced shifts in the wavenumber axis of Raman spectroscopy.

The results of the FWHM are given in Figure 3(a,b) for paracetamol and polystyrene, respectively. Similar results are shown in Figure S11 $(a, b)$ for cyclohexane and the NeAr lamp, respectively. The cross-setup variations are clearly seen from all results. In addition, the setup 'ID22' was detected as an 'outlier', having significantly larger FWHM (i.e., lower spectral resolution), which turned out to differ from others as it was a fibre-optics based setup with low nominal spectral resolution (see Table S1). In addition, the intensity calibration, which was undertaken in some setups, did not significantly influence the peak widths. This can be seen from the similar results for all the encircled pairs in the plots, which correspond to the results from spectra of the same setup, with and without the intensity calibration.

To check the influence of nominal spectral resolution (i.e., pixel size in wavenumber) on the estimation of the peak shift and peak width, we visualized additionally the results of the peak shift and the FWHM from all setups with respect to their nominal spectral resolutions. As shown in Figures S12,13, no correlation can be concluded between the spectral resolution and the peak shift or peak width. This justifies our estimation of these two spectral characteristics, which were not systematically biased by the differences in the nominal spectral resolution (i.e., pixel size).

The results of the peak ratios are given in Figure 4 and Figure S14. The curved brown arrows indicate data pairs from the same setup, with (ical) and without (raw) intensity calibration. In 
general, the peak ratios varied largely across setups. Additionally, there was no clear trend as to how the peak ratios change with the source wavelengths. Rather, however, the intensity calibration did not necessarily bring the peak ratios closer to the global mean; i.e., this correction did not improve the crosssetup comparability. In contrast, the influence of the intensity calibration seemed to be rather arbitrary. These facts very likely indicate the inaccuracy of the estimated intensity response function.

For clearer interpretation, we additionally summarized the statistics of the peak ratios in Table 3, corresponding to the results of the three source wavelengths: 514,532 , and $785 \mathrm{~nm}$. Therein, $\bar{r}$ and $s d_{0}$ represent the mean and standard deviation of each setup, while mean $(\bar{r})$ and $s d$ represent the global mean and standard deviation. Taking mean $(\bar{r})$ as an approximation of the 'true' peak ratio, the cross- and within-setup variations can be benchmarked by $\max \left(s d_{0}\right) / \operatorname{mean}(\bar{r})$ and $s d /$ mean $(\bar{r})$, respectively. On this basis, a comparison among the three ratios showed that the cross- and within-setup variations increased when the two peaks involved are further apart in the spectra. That is to say, the variations in the relative peak intensities tend to increase between two points with longer distance along the wavenumber axis. This is understandable, as the spectrometer response function has a stronger influence if the two peaks are further apart.

With all the results shown above, we would like to stress the profound influence of the setup-dependence in Raman spectroscopy. The routinely applied spectrometer calibration clearly does not remove all setup-related effects on Raman spectra when the same chemical substance is measured on different Raman platforms. Inadequate calibration can be for multiple reasons: (i) it is difficult to duplicate the measurement conditions exactly between the standard material and real samples; (ii) Raman spectra can be angle and relative orientation dependent if the standard is crystalline, and the setup (laser/grating) is polarization sensitive; (iii) there may be influence from contaminations if the standard material is not refreshed regularly - for this particular experiment, the latter reason is clearly not the case, as one laboratory supplied all materials for analysis to all participating laboratories; (iv) the full technical details of builtin (automatic) calibrations are normally inaccessible, and thus, it is hard to ensure that exactly the same calibration methods are used by the different manufacturers or setups. The application of different calibration algorithms certainly constitutes an additional source of setup-dependence.

Based on all facts and reasons stated above, computational strategies to remove the setup-induced spectral variations are urgently needed. As a first attempt to solve this issue, we have developed recently model transfer methods to deal with crosssetup variations in Raman spectroscopy. ${ }^{26,35,36}$ However, a longterm effort is needed to completely solve the issue of setupdependence, and this can only be achieved if cross-laboratory comparisons are undertaken, such as those reported in the present study and indeed elsewhere for surface-enhanced Raman scattering. ${ }^{37}$

\section{CONCLUSIONS}

This contribution reports a large-scale and cross-laboratory round robin Raman experiment designed by researchers from approximately 50 institutes in Europe. The data were submitted by 15 different institutes and measured with 35 Raman setups, which were from five different manufacturers and configured with multiple laser sources. We have presented the results with respect to four key metrics to assess Raman performance across setups. These include the SNR, the peak shift, the peak width, and the peak ratio for spectra of a set of five common substances measured on different setups. In this way, we could quantify and analyze the cross-setup comparability and variability in Raman spectroscopy, and we could verify the appropriateness to implement spectrometer calibration. More inspiring studies and efforts are certainly needed to standardize Raman spectroscopy and hence push Raman techniques closer to realworld applications. A detailed investigation on each of the different sources that harms the spectral reproducibility can be a good start. In addition, we recommend the following actions, which we believe are worthy for future investigations.

- First, we suggest that the manufacturers make spectrometer calibration a by-default included module and make the full technical details of the correction explicit and open access. This built-in process is, on the one hand, more likely to achieve 'duplicated' conditions for the real samples and the standard material and, on the other hand, makes calibration easier. In addition, by applying the same calibration procedure, the variations introduced by inhouse written calibration programs in different labs are reduced. Moreover, further investigations of the temporal reliability of instruments and calibration are needed. Any short-term setup instabilities should be understood and well controlled for the development of clinical applications.

- Second, we recommend that the manufacturers provide access to the real raw data, before any processing steps are applied, so that it is more feasible to unravel data that are closer to the 'physical truth'. We would like to encourage both researchers and manufacturers to work together on standard operating procedures for verifying instrument calibration and performance.

- Third, we encourage researchers and scientists to make their data openly available and actively contribute to establishing larger databases. This has been done in other data-rich communities (e.g., ref $^{38}$ ) and would be a vast invaluable resource to build statistical models that are tolerant to unwanted spectral differences, like the spectral variations between seemingly identical analytical setups. In this way, reproducible predictions for different measurements from the same sample can be achieved. A larger database can also provide a better reference used for the spectral alignment approaches.

- Finally, we advocate a broader cooperation on the same scientific question in order to come up with 'global' solutions and reduce the variations in the setups used in different research groups answering the same question.

\section{ASSOCIATED CONTENT}

\section{Supporting Information}

The Supporting Information is available free of charge at https://pubs.acs.org/doi/10.1021/acs.analchem.0c02696.

(Table S1) Acceptable signal-to-noise ratio for specific band integral intensities across the at least 10 submitted spectra; (Table S2) information of Raman spectroscopic devices within the trial; (Figure S1) error in the measurement of the setup ID25; (Figure S2) mean spectra and standard deviation spectra of agar; (Figure S3) mean spectra and standard deviation spectra of gelatine; (Figure S4) mean and standard deviation spectra 
and the representative Raman bands of paracetamol; (Figure S5) mean and standard deviation spectra and the representative Raman bands of polystyrene; (Figure S6) mean and standard deviation spectra and the representative Raman bands of cyclohexane; (Figure S7) mean spectra and standard deviation spectra of the NeAr lamp; (Figure S8) example of estimated SNR; (Figure S9) results of SNR for agar and gelatine; (Figure S10) results of peak shifts for cyclohexane without and with wavenumber calibration; (Figure S11) results of FWHM calculated from cyclohexane and NeAr; (Figure S12) mean absolute deviation with respective to the nominal spectral resolution of different setups; (Figure S13) FWHM with respective to the nominal spectral resolution; and (Figure S14) results of the peak ratio between the cyclohexane peaks at 1444 and $801 \mathrm{~cm}^{-1}$ (PDF)

\section{AUTHOR INFORMATION}

\section{Corresponding Author}

Thomas Bocklitz - Institute of Physical Chemistry and Abbe Center of Photonics, University Jena, 07743 Jena, Germany; Member of Leibniz Health Technologies, Leibniz Institute of Photonic Technology Jena, 07745 Jena, Germany;

() orcid.org/0000-0003-2778-6624;

Email: thomas.bocklitz@uni-jena.de

\section{Authors}

Shuxia Guo - Institute of Physical Chemistry and Abbe Center of Photonics, University Jena, 07743 Jena, Germany; Member of Leibniz Health Technologies, Leibniz Institute of Photonic Technology Jena, 07745 Jena, Germany; $\odot$ orcid.org/00000001-8237-8936

Claudia Beleites - Member of Leibniz Health Technologies, Leibniz Institute of Photonic Technology Jena, 07745 Jena, Germany; Chemometrix GmbH, 61200 Wölfersheim, Germany

Ute Neugebauer - Institute of Physical Chemistry and Abbe Center of Photonics, University Jena, 07743 Jena, Germany; Member of Leibniz Health Technologies, Leibniz Institute of Photonic Technology Jena, 07745 Jena, Germany; Center for Sepsis Control and Care, Jena University Hospital, D-07747 Jena, Germany

Sara Abalde-Cela - International Iberian Nanotechnology Laboratory (INL), 4715-310 Braga, Portugal; 10 orcid.org/ 0000-0001-5615-594X

Nils Kristian Afseth - Nofima - Norwegian Institute of Food, Fisheries and Aquaculture Research, NO-9291 Tromsø, Norway

Fatima Alsamad - Université de Reims Champagne-Ardenne, Reims 51097, France

Suresh Anand - National Institute of Optics, National Research Council, 50019 Sesto Fiorentino, Italy

Cuauhtemoc Araujo-Andrade - ICFO-Institut de Ciencies Fotoniques, The Barcelona Institute of Science and Technology, 08860 Barcelona, Spain

Sonja Aškrabić - Institute of Physics Belgrade, University of Belgrade, Beograd, Serbia

Ertug Avci - Genetics and Bioengineering Department, Faculty of Engineering, Yeditepe University, Kayisdagi 34755, Ataşehir/İstanbul, Turkey

Monica Baia - Faculty of Physics, Babes-Bolyai University, ClujNapoca 400084, Romania
Malgorzata Baranska - Faculty of Chemistry, Jagiellonian University, 30-387 Krakow, Poland; Jagiellonian Centre for Experimental Therapeutics (JCET), 30-348 Kraków, Poland; (1) orcid.org/0000-0001-8826-3144

Enrico Baria - Department of Physics, University of Florence, 50121 Firenze, FIorence, Italy; European Laboratory for Nonlinear Spectroscopy, 50019 Sesto Fiorentino, FIorence, Italy

Luis A. E. Batista de Carvalho - Molecular Physical Chemistry ReD Unit, Department of Chemistry, University of Coimbra, 3004-535 Coimbra, Portugal; 이이이.org/0000-00028059-8537

Philippe de Bettignies - HORIBA France SAS, 59650 Villeneuve-d'Ascq, France

Alois Bonifacio - Raman Lab, Dept. Engineering and Architecture, University of Trieste, 34127 Trieste, Italy; (1) orcid.org/0000-0002-2251-7786

Franck Bonnier - Faculty of pharmacy, EA6295 NanoMédicaments et Nanosondes, University of Tours, 37000 Tours, France

Eva Maria Brauchle - NMI Natural and Medical Sciences Institute at the University of Tübingen, 72770 Reutlingen, Germany; Department of Women's Health, Research Institute of Women's Health and Cluster of Excellence iFIT (EXC 2180) "Image-Guided and Functionally Instructed Tumor Therapies", Eberhard Karls University Tübingen, 72074 Tübingen, Germany

Hugh J. Byrne - FOCAS Research Institute, Technological University Dublin, Dublin, Ireland

Igor Chourpa - Faculty of pharmacy, EA6295 NanoMédicaments et Nanosondes, University of Tours, 37000 Tours, France

Riccardo Cicchi - National Institute of Optics, National Research Council, 50019 Sesto Fiorentino, Italy; European Laboratory for Non-linear Spectroscopy, 50019 Sesto Fiorentino, FIorence, Italy

Frederic Cuisinier - LBN, University Montpellier, 34000 Montpellier, France

Mustafa Culha - Genetics and Bioengineering Department, Faculty of Engineering, Yeditepe University, Kayisdagi 34755, Ataşehir/İstanbul, Turkey

Marcel Dahms - Institute of Physical Chemistry and Abbe Center of Photonics, University Jena, 07743 Jena, Germany; Member of Leibniz Health Technologies, Leibniz Institute of Photonic Technology Jena, 07745 Jena, Germany; Center for Sepsis Control and Care, Jena University Hospital, D-07747 Jena, Germany

Catalina David - HORIBA France SAS, 59650 Villeneuved'Ascq, France

Ludovic Duponchel - LASIRE - LAboratoire de Spectroscopie pour les Interactions, la Réactivité et l'Environnement, Univ. Lille, CNRS, F-59000 Lille, France; ํㅏㅇㅣ.org/0000-00027206-4498

Shiyamala Duraipandian - FOCAS Research Institute and School of Physics \& Clinical \& Optometric Sciences, Technological University Dublin, Dublin, Ireland

Samir F. El-Mashtoly - Center for Protein Diagnostics (ProDi) and Department of Biophysics, Faculty of Biology and Biotechnology, Ruhr University Bochum, 44801 Bochum, Germany; 10 orcid.org/0000-0001-6087-8817

David I. Ellis - Manchester Institute of Biotechnology, School of Chemistry, University of Manchester, M1 7DN Manchester, United Kingdom; (1) orcid.org/0000-0002-7633-7019 
Gauthier Eppe - Mass Spectrometry Laboratory, MolSys Research Unit, University of Liege, 4000 Liège, Belgium

Guillaume Falgayrac - MABLab, Marrow Adiposity and Bone Lab, Univ. Littoral Côte d'Opale, F-62300 Boulogne-sur-Mer, France; CHU Lille, F-59000 Lille, France; 이이.org/ 0000-0003-1806-1509

Ozren Gamulin - Department of Physics and Biophysics, School of Medicine, University of Zagreb, 10000 Zagreb, Croatia; Centre for Advanced Materials Science, 10000 Zagreb, Croatia

Benjamin Gardner - Physics and Astronomy, Mathematics and Physical Sciences, College of Engineering, Exeter EX4 4Q, United Kingdom; (1) orcid.org/0000-0002-7223-9585

Peter Gardner - Manchester Institute of Biotechnology, School of Chemistry, University of Manchester, M1 7DN Manchester, United Kingdom; Department of Chemical Engineering and Analytical Science, School of Engineering, The University of Manchester, Manchester M1 3AL, United Kingdom; (1) orcid.org/0000-0003-4034-3764

Klaus Gerwert - Center for Protein Diagnostics (ProDi) and Department of Biophysics, Faculty of Biology and Biotechnology, Ruhr University Bochum, 44801 Bochum, Germany

Evangelos J. Giamarellos-Bourboulis - 4th Department of Internal Medicine, National and Kapodistrian University of Athens, Medical School, Athens, Greece

Sveinbjorn Gizurarson - Faculty of Pharmaceutical Sciences, University of Iceland, Reykjavik, Iceland

Marcin Gnyba - Faculty of Electronics, Telecommunications and Informatics, Gdańsk University of Technology, 80-233 Gdańsk, Poland

Royston Goodacre - Department of Biochemistry and Systems Biology, Institute of Systems, Molecular and Integrative Biology, University of Liverpool, Liverpool L69 750 7ZB, United Kingdom

Patrick Grysan - Materials Research and Technology, Luxembourg Institute of Science and Technology, L-4422 Belvaux, Luxembourg

Orlando Guntinas-Lichius - Department of Otorhinolaryngology, Jena University Hospital, 07743 Jena, Germany; 이이.orcid.org/0000-0001-9671-0784

Helga Helgadottir - Faculty of Pharmaceutical Sciences, University of Iceland, Reykjavik, Iceland

Vlasta Mohaček Grošev - Centre for Advanced Materials Science, 10000 Zagreb, Croatia; Ruđer Bošković Institute, 10000 Zagreb, Croatia

Catherine Kendall - Biophotonics Research Unit, Gloucestershire Hospitals NHS Foundation Trust, Gloucester GL1 3NN, United Kingdom

Roman Kiselev - Member of Leibniz Health Technologies, Leibniz Institute of Photonic Technology Jena, 07745 Jena, Germany; St. Jude Children's Research Hospital, Memphis, Tennessee 38105, United States

Micha Kölbach - Renishaw GmbH, 72124 Pliezhausen, Germany

Christoph Krafft - Member of Leibniz Health Technologies, Leibniz Institute of Photonic Technology Jena, 07745 Jena, Germany

Sivashankar Krishnamoorthy - Materials Research and Technology, Luxembourg Institute of Science and Technology, L-4422 Belvaux, Luxembourg; (1) orcid.org/0000-00030410-1696

Patrick Kubryck - Renishaw GmbH, 72124 Pliezhausen, Germany
Bernhard Lendl - Institute of Chemical Technologies and Analytics, TU Wien, 1040 Wien, Austria; (1) orcid.org/00000003-3838-5842

Pablo Loza-Alvarez - ICFO-Institut de Ciencies Fotoniques, The Barcelona Institute of Science and Technology, 08860 Barcelona, Spain

Fiona M. Lyng - FOCAS Research Institute and School of Physics \& Clinical \& Optometric Sciences, Technological University Dublin, Dublin, Ireland

Susanne Machill - Chair of Bioanalytical Chemistry, TU Dresden, 01062 Dresden, Germany

Cedric Malherbe - Mass Spectrometry Laboratory, MolSys Research Unit, University of Liege, 4000 Liège, Belgium

Monica Marro - ICFO-Institut de Ciencies Fotoniques, The Barcelona Institute of Science and Technology, 08860 Barcelona, Spain; 10 orcid.org/0000-0003-4031-4605

Maria Paula M. Marques - Molecular Physical Chemistry R\&D Unit, Department of Chemistry and Department of Life Sciences, University of Coimbra, 3004-535 Coimbra, Portugal; $\odot$ orcid.org/0000-0002-8391-0055

Ewelina Matuszyk - Jagiellonian Centre for Experimental Therapeutics (JCET), 30-348 Kraków, Poland

Carlo Francesco Morasso - Istituti Clinici Scientifici Maugeri IRCCS, 27100 Pavia, Italy

Myriam Moreau - LASIRE - LAboratoire de Spectroscopie pour les Interactions, la Réactivité et l'Environnement, Univ. Lille, CNRS, F-59000 Lille, France

Howbeer Muhamadali - Department of Biochemistry and Systems Biology, Institute of Systems, Molecular and Integrative Biology, University of Liverpool, Liverpool L69 750 7ZB, United Kingdom

Valentina Mussi - National Research Council, Institute for Microelectronics and Microsystems (IMM-CNR), 00133 Rome, Italy; (1) orcid.org/0000-0003-1465-1739

Ioan Notingher - School of Physics and Astronomy, University of Nottingham, Nottingham NG7 2RD, United Kingdom; (1) orcid.org/0000-0002-5360-230X

Marta Z. Pacia - Jagiellonian Centre for Experimental Therapeutics (JCET), 30-348 Kraków, Poland; ํorcid.org/ 0000-0002-0391-2502

Francesco S. Pavone - Department of Physics, University of Florence, 50121 Firenze, FIorence, Italy; European Laboratory for Non-linear Spectroscopy, 50019 Sesto Fiorentino, FIorence, Italy

Guillaume Penel - MABLab, Marrow Adiposity and Bone Lab, Univ. Littoral Côte d'Opale, F-62300 Boulogne-sur-Mer, France; CHU Lille, F-59000 Lille, France

Dennis Petersen - Department of Biophysics, Faculty of Biology and Biotechnology, Ruhr University Bochum, 44801 Bochum, Germany

Olivier Piot - Université de Reims Champagne-Ardenne, Reims 51097, France

Julietta V. Rau - Istituto di Struttura della Materia, Consiglio Nazionale delle Ricerche (ISM-CNR), 100-00133 Rome, Italy; Sechenov First Moscow State Medical University, 119991 Moscow, Russian Federation; (1) orcid.org/00000002-7953-1853

Marc Richter - Renishaw GmbH, 72124 Pliezhausen, Germany

Maria Krystyna Rybarczyk - Chemical Faculty, Gdansk University of Technology, 80-233 Gdansk, Poland

Hamideh Salehi - LBN, University Montpellier, 34000 Montpellier, France 
Katja Schenke-Layland - NMI Natural and Medical Sciences Institute at the University of Tübingen, 72770 Reutlingen, Germany; Department of Women's Health, Research Institute of Women's Health and Cluster of Excellence iFIT (EXC 2180) "Image-Guided and Functionally Instructed Tumor Therapies", Eberhard Karls University Tübingen, 72074 Tübingen, Germany; (1) orcid.org/0000-0001-8066-5157

Sebastian Schlücker - Faculty of Chemistry, University of Duisburg-Essen, 45141 Essen, Germany; 이이이.org/00000003-4790-4616

Markus Schosserer - Department of Biotechnology, Institute of Molecular Biotechnology, University of Natural Resources and Life Sciences, 1180, Austria; 이이이.org/0000-0003-20250739

Karin Schütze - CellTool GmbH, 82327 Tutzing, Germany

Valter Sergo - Raman Lab, Dept. Engineering and Architecture, University of Trieste, 34127 Trieste, Italy; Faculty of Health Sciences, University of Macau, 999078 Macau, SAR China; (1) orcid.org/0000-0003-3864-658X

Faris Sinjab - School of Physics and Astronomy, University of Nottingham, Nottingham NG7 2RD, United Kingdom

Janusz Smulko - Faculty of Electronics, Telecommunications and Informatics, Gdańsk University of Technology, 80-233 Gdańsk, Poland; (1) orcid.org/0000-0003-1459-4199

Ganesh D. Sockalingum - Université de Reims ChampagneArdenne, Reims 51097, France

Clara Stiebing - Member of Leibniz Health Technologies, Leibniz Institute of Photonic Technology Jena, 07745 Jena, Germany

Nick Stone - Physics and Astronomy, Mathematics and Physical Sciences, College of Engineering, Exeter EX4 4Q, United Kingdom; 이이.org/0000-0001-5603-3731

Valérie Untereiner - Université de Reims ChampagneArdenne, 51097 Reims, France

Renzo Vanna - Istituti Clinici Scientifici Maugeri IRCCS, 27100 Pavia, Italy

Karin Wieland - Institute of Chemical Technologies and Analytics, TU Wien, 1040 Wien, Austria

Jürgen Popp - Institute of Physical Chemistry and Abbe Center of Photonics, University Jena, 07743 Jena, Germany; Member of Leibniz Health Technologies, Leibniz Institute of Photonic Technology Jena, 07745 Jena, Germany

Complete contact information is available at:

https://pubs.acs.org/10.1021/acs.analchem.0c02696

\section{Notes}

The authors declare no competing financial interest.

\section{ACKNOWLEDGMENTS}

This publication is based upon work from COST Action BM1401 Raman4Clinics, supported by COST (European Cooperation in Science and Technology). COST is a funding agency for research and innovation networks (www.cost.eu). Our actions help connect research initiatives across Europe and enable scientists to grow their ideas by sharing them with their peers. This boosts their research, career, and innovation. The authors would like to thank M.D. for preparing gelatine and agar gels. R.K. portioned, packaged, and shipped the samples with the help of C.S. and M. Spreemann who did the customs declarations for samples shipped outside the EU. The authors highly thank M. Bedoni, L. Colombo, H. Dehghani, A. Mourka, W. Nahm, A. Pifferi, and L. Spinelli, who have actively contributed to the parallel discussion session 'Open Data' at the workshop 'Performance Assessment and Standardization in Biophotonics' that took place on 12.09.2019 in Brussels. The ideas and suggestions listed in the conclusion of this manuscript include a collective contribution from this parallel discussion. L.A.B.C. and M.P.M.M. acknowledge the Portuguese Foundation for Science and Technology (Project UIDB/00070/2020). S.K. and P.G. acknowledge the funding from National Research Fund of Luxembourg (FNR) via the project PLASENS (C15/ MS/10459961). The scholarship from the China scholarship council (CSC) for S.G is highly acknowledged. This project was supported by the BOKU Core Facilities Multiscale Imaging. Funded by the Deutsche Forschungsgemeinschaft (DFG, German Research Foundation) - 441958208.

\section{REFERENCES}

(1) Bocklitz, T. W.; Guo, S.; Ryabchykov, O.; Vogler, N.; Popp, J. r. Anal. Chem. 2015, 88, 133-151.

(2) Butler, H. J.; Ashton, L.; Bird, B.; Cinque, G.; Curtis, K.; Dorney, J.; Esmonde-White, K.; Fullwood, N. J.; Gardner, B.; Martin-Hirsch, P. L. Nat. Protoc. 2016, 11, 664.

(3) Cheng, J.-X.; Xie, X. S. Science 2015, 350, No. aaa8870.

(4) de Oliveira Penido, C. A. F.; Pacheco, M. T. T.; Lednev, I. K.; Silveira, L., Jr. J. Raman Spectrosc. 2016, 47, 28-38.

(5) Doty, K. C.; Lednev, I. K. TrAC, Trends Anal. Chem. 2018, 103, 215-222.

(6) Marro, M.; Taubes, A.; Abernathy, A.; Balint, S.; Moreno, B.; Sanchez-Dalmau, B.; Martínez-Lapiscina, E. H.; Amat-Roldan, I.; Petrov, D.; Villoslada, P. J. Biophoton. 2014, 7, 724-734.

(7) Neugebauer, U.; Bocklitz, T.; Clement, J.; Krafft, C.; Popp, J. Analyst 2010, 135, 3178-3182.

(8) Stremersch, S.; Marro, M.; Pinchasik, B. E.; Baatsen, P.; Hendrix, A.; De Smedt, S. C.; Loza-Alvarez, P.; Skirtach, A. G.; Raemdonck, K.; Braeckmans, K. Small 2016, 12, 3292-3301.

(9) Vogler, N.; Bocklitz, T.; Subhi Salah, F.; Schmidt, C.; Bräuer, R.; Cui, T.; Mireskandari, M.; Greten, F. R.; Schmitt, M.; Stallmach, A. J. biophotonics 2016, 9, 533-541.

(10) Aljakouch, K.; Lechtonen, T.; Yosef, H. K.; Hammoud, M. K.; Alsaidi, W.; Kötting, C.; Mügge, C.; Kourist, R.; El-Mashtoly, S. F.; Gerwert, K. Angew. Chem., Int. Ed. 2018, 57, 7250-7254.

(11) Marro, M.; Nieva, C.; de Juan, A.; Sierra, A. Anal. Chem. 2018, 90, $5594-5602$.

(12) Van Nest, S. J.; Nicholson, L. M.; Pavey, N.; Hindi, M. N.; Brolo, A. G.; Jirasek, A.; Lum, J. J. BMC cancer 2019, 19, 474.

(13) Hanson, C.; Barney, J. T.; Bishop, M. M.; Vargis, E. Electrophoresis 2019, 40, 1446-1456.

(14) Stöckel, S.; Meisel, S.; Elschner, M.; Rosch, P.; Popp, J. Anal. Chem. 2012, 84, 9873-9880.

(15) Enejder, A. M.; Koo, T.-W.; Oh, J.; Hunter, M.; Sasic, S.; Feld, M. S.; Horowitz, G. L. Opt. Lett. 2002, 27, 2004-2006.

(16) Schut, T. C. B.; Puppels, G. J.; Kraan, Y. M.; Greve, J.; Van Der Maas, L. L.; Figdor, C. G. Int. J. Cancer 1997, 74, 20-25.

(17) Zakel, S.; Rienitz, O.; Güttler, B.; Stosch, R. Analyst 2011, 136, 3956-3961.

(18) Jiang, Y.; Sun, D.-W.; Pu, H.; Wei, Q. Trends Food Sci. Technol. 2018, 75, 10-22.

(19) Su, W. H.; Sun, D. W. Compr. Rev. Food Sci. Food Saf. 2018, 17, 104-122.

(20) McCreery, R. L., Raman spectroscopy for chemical analysis. John Wiley \& Sons: 2005; Vol. 225.

(21) Bocklitz, T.; Dörfer, T.; Heinke, R.; Schmitt, M.; Popp, J. Spectrochim. Acta, Part A 2015, 149, 544-549.

(22) Dörfer, T.; Bocklitz, T.; Tarcea, N.; Schmitt, M.; Popp, J. Zeitsc. für Phys. Chem. 2011, 225, 753-764.

(23) Mann, C. K.; Vickers, T. J. Appl. Spectrosc. 2016, 53, 856-861.

(24) Bloemberg, T. G.; Gerretzen, J.; Lunshof, A.; Wehrens, R.; Buydens, L. M. Anal. Chim. Acta 2013, 781, 14-32. 
(25) Afseth, N. K.; Kohler, A. Chemom. Intell. Lab. Syst. 2012, 117, 92-99.

(26) Guo, S.; Kohler, A.; Zimmermann, B.; Heinke, R.; Stöckel, S.; Rösch, P.; Popp, J. r.; Bocklitz, T. Anal. Chem. 2018, 90, 9787-9795.

(27) Smilde, A. K.; Jansen, J. J.; Hoefsloot, H. C.; Lamers, R.-J. A.; Van Der Greef, J.; Timmerman, M. E. Bioinformatics 2005, 21, 3043-3048.

(28) Agency E. M., Guideline on bioanalytical method validation. 21 July 2011.

(29) Miroslav, M., Peaks: Peaks. 2012.

(30) Press, W. H.; Teukolsky, S. A. Comp. in Phys. 1990, 4, 669-672.

(31) Bradley, M., Curve fitting in Raman and IR spectroscopy: basic theory of line shapes and applications. Thermo Fisher Scientific, Madison, USA; Application Note 2007, 50733.

(32) E1840-96, A, Standard guide for Raman shift standards for spectrometer calibration. ASTM International: West Conshohocken, PA; 2014.

(33) E2529-06, A, Standard Guide for Testing the Resolution of a Raman Spectromete. ASTM International: West Conshohocken, PA, 2014.

(34) E2911-13, A, Standard Guide for Relative Intensity Correction of Raman Spectrometers. ASTM International: West Conshohocken, PA, 2013.

(35) Guo, S.; Heinke, R.; Stöckel, S.; Rösch, P.; Bocklitz, T.; Popp, J. Vib. Spectrosc. 2017, 91, 111-118.

(36) Guo, S.; Heinke, R.; Stöckel, S.; Rösch, P.; Popp, J.; Bocklitz, T. J. Raman Spectrosc. 2018, 49, 627-637.

(37) Fornasaro, S.; Alsamad, F.; Baia, M.; Batista de Carvalho, L. A. E.; Beleites, C.; Byrne, H. J.; Chiadò, A.; Chis, M.; Chisanga, M.; Daniel, A. Anal. Chem. 2020, 92, 4053-4064.

(38) Salek, R. M.; Neumann, S.; Schober, D.; Hummel, J.; Billiau, K.; Kopka, J.; Correa, E.; Reijmers, T.; Rosato, A.; Tenori, L. Metabolomics 2015, 11, 1587-1597. 